BMC

Genomics
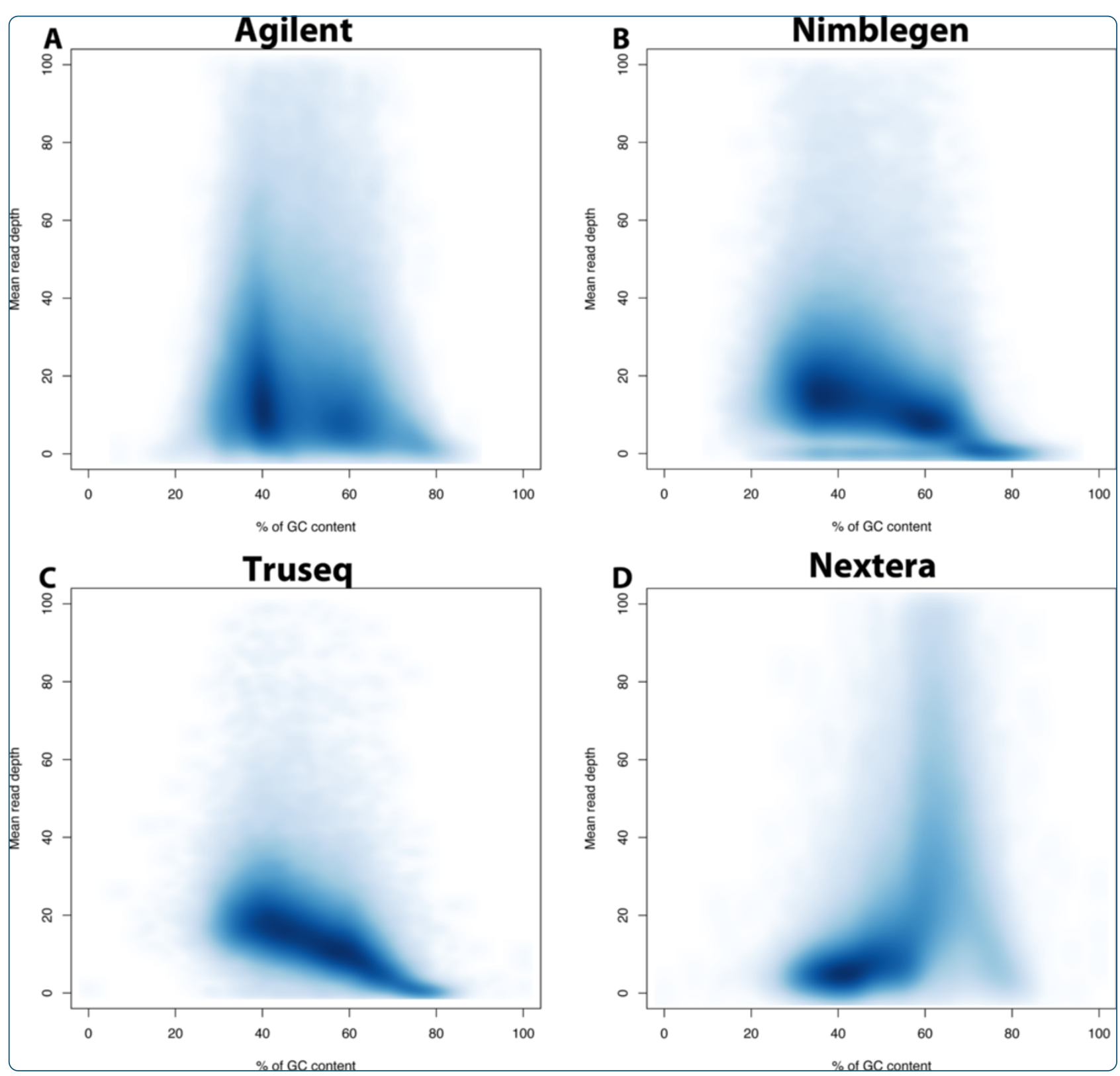

\title{
Performance comparison of four exome capture systems for deep sequencing
}

Chilamakuri et al. 


\title{
Performance comparison of four exome capture systems for deep sequencing
}

\author{
Chandra Sekhar Reddy Chilamakuri ${ }^{1,3^{*}}$, Susanne Lorenz ${ }^{1,3,4}$, Mohammed-Amin Madoui ${ }^{1,4}$, Daniel Vodák ${ }^{1,5}$, \\ Jinchang Sun ${ }^{1,3,4}$, Eivind Hovig ${ }^{1,2,3,5}$, Ola Myklebost ${ }^{1,3}$ and Leonardo A Meza-Zepeda ${ }^{1,3,4^{*}}$
}

\begin{abstract}
Background: Recent developments in deep (next-generation) sequencing technologies are significantly impacting medical research. The global analysis of protein coding regions in genomes of interest by whole exome sequencing is a widely used application. Many technologies for exome capture are commercially available; here we compare the performance of four of them: NimbleGen's SeqCap EZ v3.0, Agilent's SureSelect v4.0, Illumina's TruSeq Exome, and Illumina's Nextera Exome, all applied to the same human tumor DNA sample.
\end{abstract}

Results: Each capture technology was evaluated for its coverage of different exome databases, target coverage efficiency, GC bias, sensitivity in single nucleotide variant detection, sensitivity in small indel detection, and technical reproducibility. In general, all technologies performed well; however, our data demonstrated small, but consistent differences between the four capture technologies. Illumina technologies cover more bases in coding and untranslated regions. Furthermore, whereas most of the technologies provide reduced coverage in regions with low or high GC content, the Nextera technology tends to bias towards target regions with high GC content.

Conclusions: We show key differences in performance between the four technologies. Our data should help researchers who are planning exome sequencing to select appropriate exome capture technology for their particular application.

Keywords: Exome capture technology, Next-generation sequencing, Coverage efficiency, Enrichment efficiency, GC bias, Single nucleotide variant, Indel

\section{Background}

In general it remains prohibitively expensive to analyze whole genomes for population scale study, even though the cost of whole genome sequencing has fallen significantly [1]. As an alternative, the targeted resequencing of subsets of a genome is more feasible. The most widely used approach captures much of the entire protein coding region of a genome (the exome), which makes up about $1 \%$ of the human genome, and has become a routine technique in clinical and basic research [2-5]. Exome sequencing offers definite advantages over whole genome sequencing: it is significantly less expensive, more easily understood for functional interpretation, significantly faster to analyze, and

\footnotetext{
* Correspondence: chichi@rr-research.no; Leonardo.Meza-Zepeda@rr-research.no 'Department of Tumor Biology, Oslo University Hospital, Norwegian Radium Hospital, 0310 Oslo, Norway

${ }^{3}$ Norwegian Cancer Genomics Consortium, Oslo, Norway

Full list of author information is available at the end of the article
}

an easy dataset to manage. Multiple technologies have surfaced for the enrichment of target regions of interest, as the demand for targeted resequencing has increased over time. Broadly, these technologies can be classified into two groups, chip-based exome capture versus solution-based exome capture. Chip-based exome capture was the first to be developed [6,7], but required large amounts of input DNA, and was quickly replaced by more efficient solutionbased capture systems. There are currently four major solution-based human exome capture systems available: Agilent's SureSelect Human All Exon, NimbleGen's SeqCap EZ Exome Library [8], Illumina's TruSeq Exome Enrichment, and Illumina's Nextera Exome Enrichment [9]. Exome capture involves the capture of protein coding regions by hybridization of genomic DNA to biotinylated oligonucleotide probes (baits). These technologies use biotinylated DNA or RNA baits complementary to targeted exons, which are hybridized to genomic fragment libraries. Magnetic streptavidin beads are used to selectively pull- 
down and enrich baits with bound targeted regions. The sample preparation methods are highly similar across the different technologies. The major differences between the technologies correspond to the choice of their respective target regions, bait lengths, bait density, molecules used for capture, and genome fragmentation method (Table 1).

Clark et al. compared three capture technologies and showed that NimbleGen technology required the least number of reads to sensitively detect small variants, whereas Agilent and Illumina technologies appeared to detect a higher total number of variants with additional reads [10]. In another study, Sulonen et al. compared NimbleGen and Agilent technologies, and showed that there were no major differences between the two technologies, except that NimbleGen showed greater efficiency in covering the exome with a minimum of $20 \mathrm{x}$ coverage [11]. Asan et al. compared NimbleGen Sequence Capture Array, NimbleGen SeqCap EZ, and Agilent SureSelect, and showed that all three technologies achieved a similar accuracy of genotype assignment and single nucleotide polymorphism (SNP) detection, and had similar levels of reproducibility and GC bias [12]. In another exome capture comparison study, Parla et al. showed that both NimbleGen SeqCap EZ Exome Library SR and Agilent SureSelect All Exon were similar to each other in performance, and able to capture most of the human exons targeted by their probe sets. However, they failed to cover a noteworthy percentage of the exons in the consensus coding sequence database (CCDS) [13].

During the past few years, substantial updates have been made to the different capture technologies, including new content and improved probe design. For instance, NimbleGen's SeqCap EZ exome library v2.0 targets approximately $44 \mathrm{Mb}$ of genome, where as their next version EZ exome library v3.0 targets $64.1 \mathrm{Mb}$. The new Illumina Nextera capture technology has to the best of our knowledge not been tested extensively vis-à-vis other technologies.

The lack of a clear consensus from previous studies, updates in three major capture technologies, and the important new Illumina Nextera capture technology, using an entirely different strategy, motivated us to perform a detailed comparative analysis before initiating a major exome sequencing project.

We, therefore, systematically compared four exome capture technologies, NimbleGen's SeqCap EZ exome library v3.0, Agilent SureSelect Human all exon V4, Illumina TruSeq and Illumina Nextera, with respect to features such as design differences relative to coverage efficiency, GC bias, and variant discovery.

\section{Results}

Distinctive features of four exome capture technologies There are considerable differences between the four exome capture technologies, as shown in Table 1. Illumina TruSeq and Nextera technologies are identical in many characteristics, except that Nextera uses transposomes for fragmentation, whereas TruSeq fragments the DNA by ultrasonication. The Agilent technology uses RNA molecules as probes, whereas all the other technologies use DNA as probe molecules. NimbleGen presents the highest number of probes, being the only technology with an overlapping probe design, thus giving it the highest probe density technology of the four. Agilent

Table 1 Exome capture technology designs

\begin{tabular}{|c|c|c|c|c|}
\hline & NimbleGen & Agilent & Illumina TruSeq & Illumina Nextera \\
\hline Bait type & DNA & RNA & DNA & DNA \\
\hline Bait length range (bp) & NP & $114-126$ & 95 & 95 \\
\hline Median bait length (bp) & NP & 119 & 95 & 95 \\
\hline Number of baits & NP & 554,079 & 347,517 & 347,517 \\
\hline Total bait length (Mb) & NP & 66.48 & 33.01 & 33.01 \\
\hline Target length range (bp) & $59-742$ & $114-21,747$ & $2-37,917$ & $2-37,917$ \\
\hline Median target length (bp) & 171 & 200 & 135 & 135 \\
\hline Number of targets & 368,146 & 185,636 & 201071 & 201,071 \\
\hline Total target length (Mb) & 64.19 & 51.18 & 62.08 & 62.08 \\
\hline Fragmentation method & Ultrasonication & Ultrasonication & Ultrasonication & Transposomes \\
\hline Automation & ++ & ++ & ++ & +++ \\
\hline Throughput & +++ & +++ & +++ & +++ \\
\hline Flexibility & Custom available & Custom available & & Custom available \\
\hline Species & Human, mouse, 3 plant species & Human, mouse, 14 other species custom & Human & Human \\
\hline Costs & $\$ \$$ & $\$ \$$ & $\$$ & $\$$ \\
\hline
\end{tabular}

Some NimbleGen information was not provided, indicated by NP. Relative automation and throughput indicated by " + " symbols, higher number of symbols indicates easy to automate and higher throughput. Relative cost is indicated by "\$" symbol, higher "\$" symbols indicate the higher price. 
probes are non-overlapping, but lie directly adjacent to one another. On the other hand, the Illumina technologies, use a gapped probe approach. The technologies also differ in the regions they target, and in the total number of bases targeted. For instance, NimbleGen targets $64.1 \mathrm{Mb}$, Agilent targets $51.1 \mathrm{Mb}$, and TruSeq and Nextera targets $62.08 \mathrm{Mb}$ of human genome.
Interestingly, only $26.2 \mathrm{Mb}$ of the total targeted bases are common among all exome capture technologies (Figure 1A). Of the four, NimbleGen and Agilent technologies have the most in common, sharing almost $40 \mathrm{Mb}$ of targeted sequences. Illumina has 22.5 million unique target bases, followed by NimbleGen with 16.1 million bases, and Agilent with 7 million unique bases.

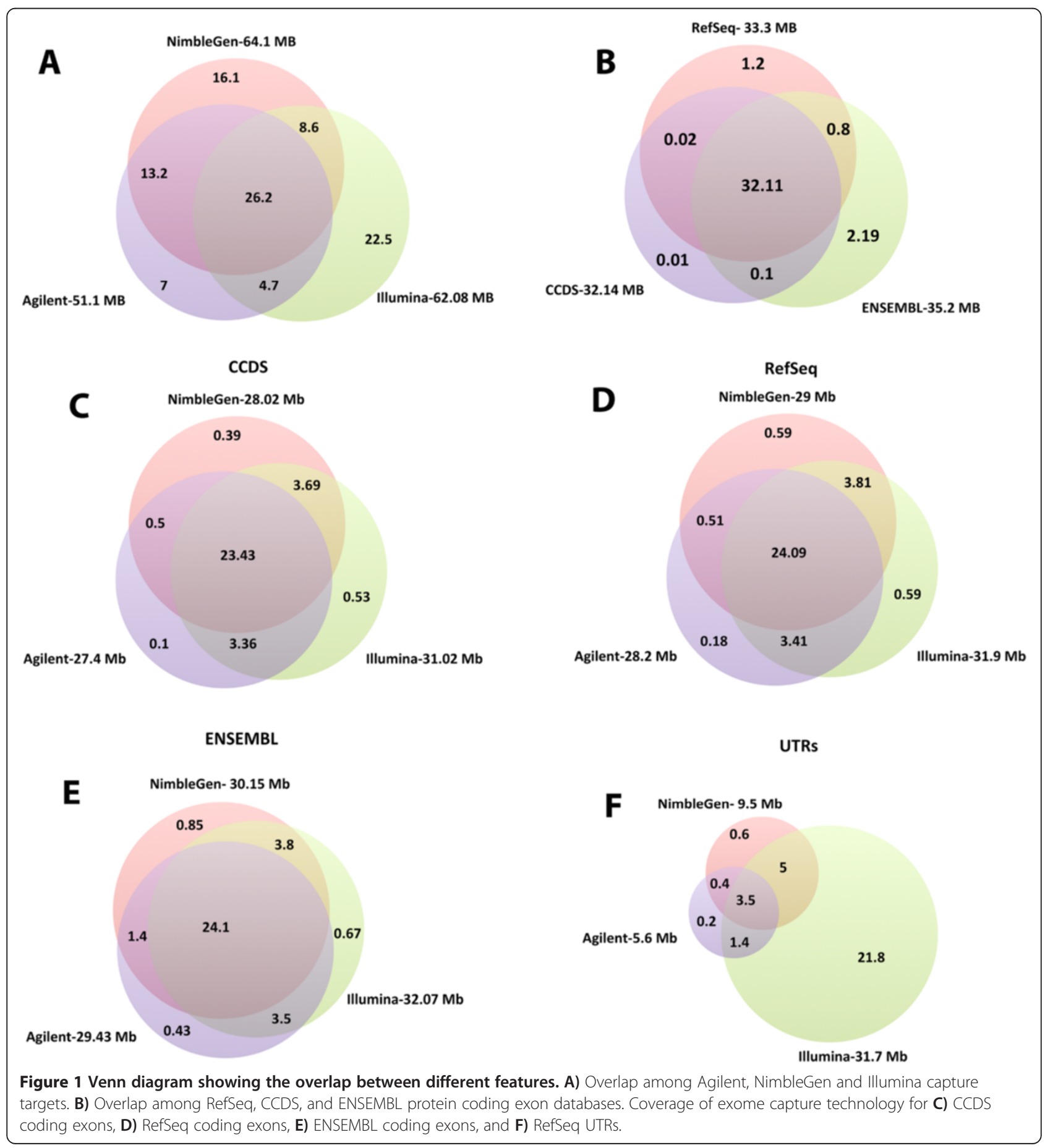


Many different RNA databases are available, such as RefSeq [14] and Ensembl [15], which differ in the number of non-coding RNAs and total number of exons reported, as well as the start and end coordinates of exons. Significant portions of the sequences are common among the different databases (Figure 1B). CCDS contains protein-coding sequences with high quality annotations [16]. RefSeq and CCDS share a greater proportion of bases with each other, whereas Ensembl possesses more unique bases (2.19 million) than the other two databases. We investigated the coverage of RefSeq (coding and UTR), Ensembl (coding) and CCDS (coding).

Illumina covers a greater portion of coding exon bases across all the databases, followed by NimbleGen and Agilent (Figure 1C-E). There are $32.11 \mathrm{Mb}$ common across the three databases, but only about $24 \mathrm{Mb}$ are covered by all four technologies. The majority of Illumina-specific bases $(22.5 \mathrm{Mb})$ target untranslated regions (UTRs) (Figure 1F), whereas NimbleGen and Agilent target UTRs at 9.5 Mb and 5.6 Mb, respectively.

\section{Sequencing, sequence alignment, and read filtering}

To evaluate each technology, two independent exome libraries derived from the tumor tissue of an osteosarcoma sample were sequenced twice (technical replicates). The exome library for each technology was prepared according to each supplier's recommended protocol. On average, 136.8 million reads were generated for each technology, varying between 95.8 and 185.1 million reads. There were also differences in sequencing and alignment rates between the different technologies. The read alignment rate varied among technologies: $97.4 \%$ for TruSeq, $97.7 \%$ for NimbleGen, 97.6\% Agilent, and $98.95 \%$ for Nextera (Figure 2A). Mapped reads from each library were further filtered for duplicates, multiple mappers, improper pairs, and off-target reads. Large variation was observed for the percentage of pass-filter mapped reads, with Agilent being the highest at $71.7 \%$ retained reads, NimbleGen next at $66.0 \%$, TruSeq at $54.8 \%$, and Nextera at $40.1 \%$ (Figure 2A). We further examined the number of reads filtered out in each of the four steps (Figure 2B). For all the technologies, the greatest number of reads lost was due to the number of reads mapped to non-targeted regions (off-target reads). Agilent showed a slightly higher percentage of off-target reads and the fewest reads mapping to multiple sites.

\section{Target coverage efficiency differs among four technologies}

We used the methods described by Clark et al. [10] to investigate target coverage efficiency. We evaluated coverage efficiency by calculating base coverage over 1) all intended target bases, 2) common bases among the four technologies, 3) Ensembl exons, 4) RefSeq exons, and 5) CCDS exons, using 50 million randomly chosen reads for each technology. Target coordinates were downloaded from the supplier's websites. It is worthwhile to note that TruSeq and Nextera, both supplied by Illumina, use the same capture baits. At this level of reads, the fractions of targets covered at least once varied somewhat, the Agilent technology captured 99.8\%, the Nextera technology captured $98.2 \%$, the TruSeq captured $96.9 \%$, and the NimbleGen captured $96.5 \%$ of the intended targets (Figure 3A). The $1 \times$ coverage number provides the fraction of the target that can potentially be covered by the respective designs. Not surprisingly, all the technologies give high coverage of their respective target regions, with the Agilent technology giving highest coverage (99.8\%). The number of intended target
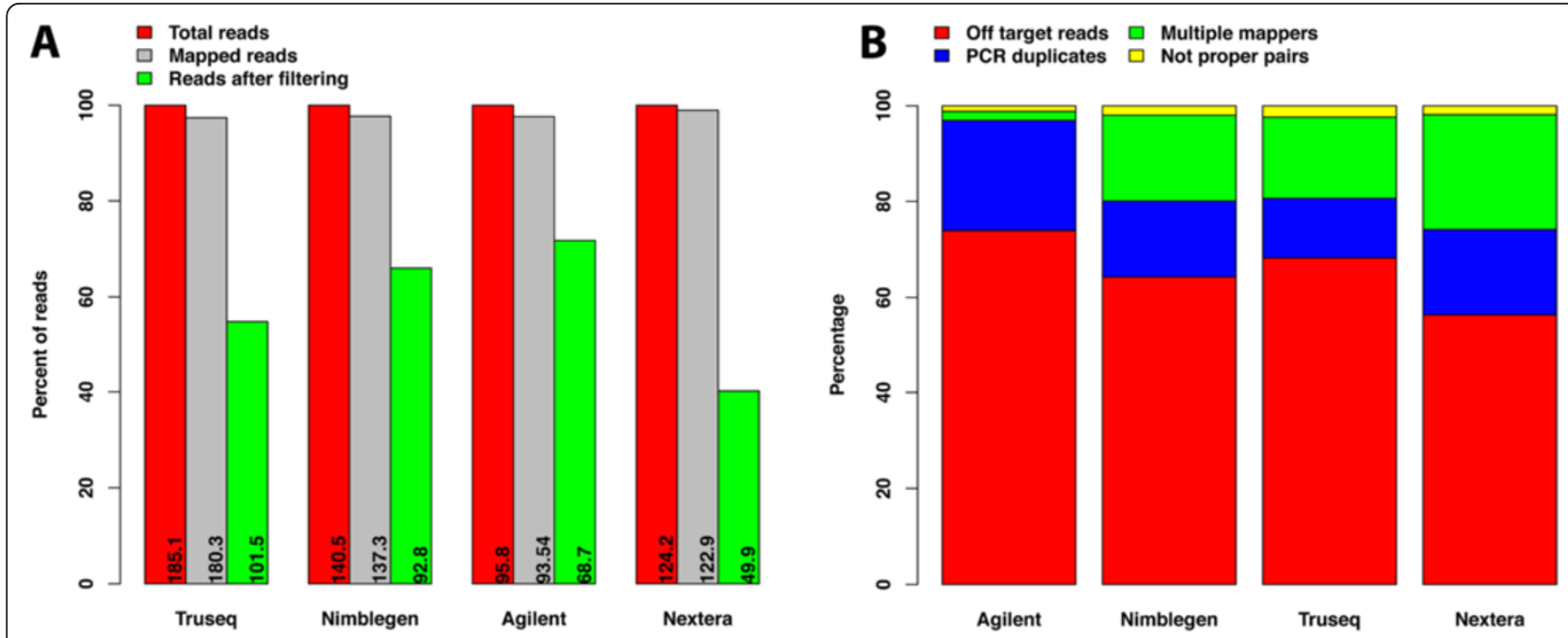

Figure 2 Read statistics. A) Bar plot showing percent of initial reads, mapped reads and reads left after filtering for four different technologies; each bar shows the number of reads in millions. B) Stacked bar plot showing subgroups of filtered reads. 

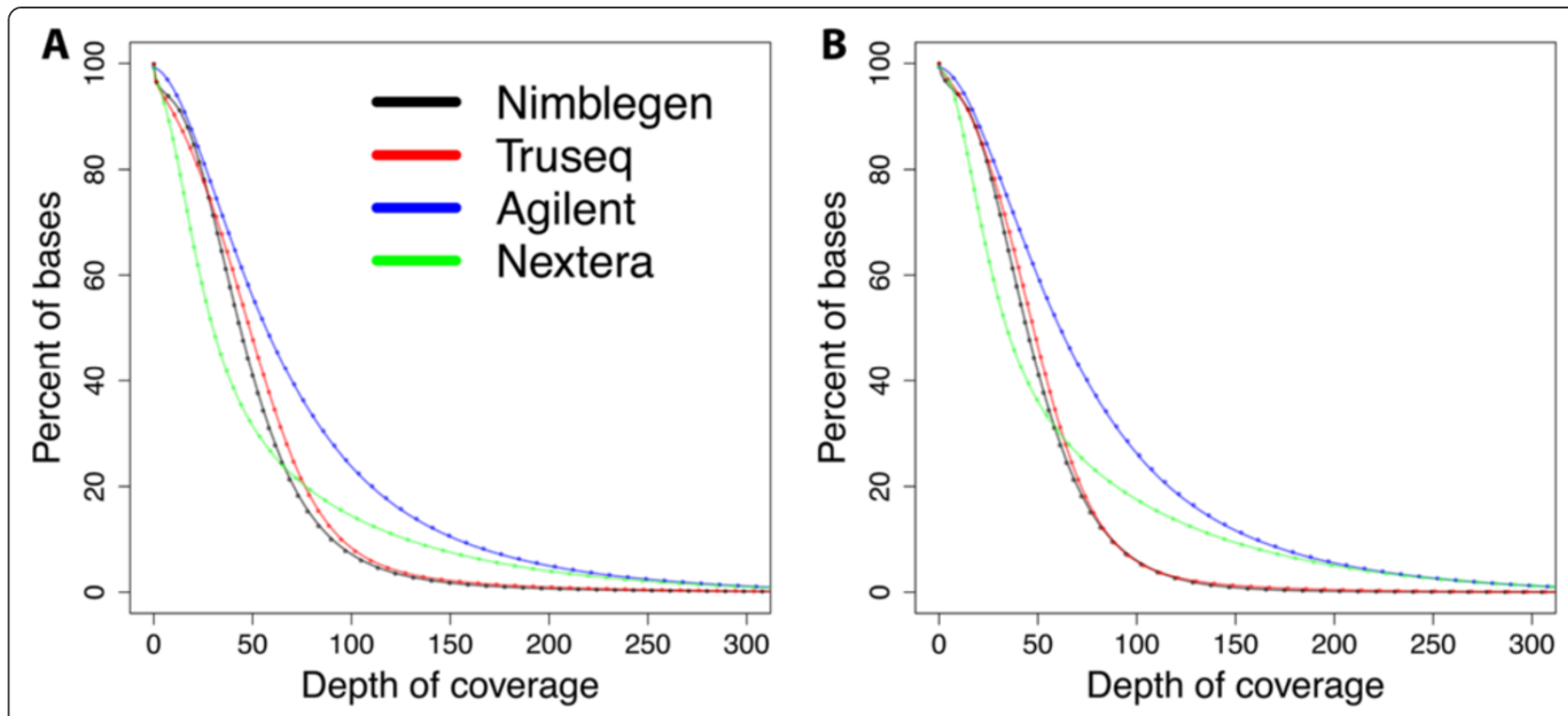

Figure 3 Coverage efficiency comparison by technology. Coverage efficiency defined as the percent of the total targeted bases covered at particular depths. A) Coverage efficiency for intended targeted bases for each technology. B) Coverage efficiency for bases, which are shared, by all four technologies (26.2 MB). Smooth line indicates replicate 1, and dotted line indicates replicate 2.

bases varies considerably, as the Agilent technology targets $51.1 \mathrm{Mb}$, NimbleGen $64.1 \mathrm{Mb}$, and Illumina $62.08 \mathrm{Mb}$ (Figure 1A), sharing only 26.2 million bases between technologies. When measured at $1 \times$ coverage on the common bases (26.2 Mb), we observed a similar trend, where the Agilent technology covers the highest number of bases, with $99.8 \%$, followed by Nextera with 99.5\%, TruSeq with $98.8 \%$, and NimbleGen with $98 \%$ (Figure 3B and Additional file 1: Figure S1). We found no major difference in coverage efficiency between two technical replicates, indicating that all four technologies give high technical reproducibility.

We next evaluated coverage efficiency as a function of sequencing depth. We randomly selected filtered reads in 5 million read increments from 5 million to 50 million. The fraction of the intended target bases, covered at depths of at least $10 \times, 20 \times, 30 \times, 40 \times, 50 \times$ and $100 \times$, was determined (Figure 4). The Agilent technology covered a higher percent of its target bases at all read counts and depth cut-offs compared with the other three technologies. For all the technologies, 25 million reads were sufficient to cover about $80 \%$ of target bases with at least $10 \times$ depth, with the exception of the Nextera technology, which covered only about $60 \%$ of target bases with the same number of reads (Figure 4A). When using 45 million reads with all the technologies, more than $80 \%$ of target bases were covered with $\geq 20 \times$ coverage, but the Nextera technology covered only $58 \%$ of the bases at the same depth (Figure 4B). For all the read counts, Agilent and Nextera covered more bases with $\geq 100 \times$ coverage than other two technologies, but showed a considerable difference in coverage (Figure 4F).

\section{Influence of GC content on coverage}

Base composition has been shown to bias sequencing efficiency, thus coverage may be low for sequences with high GC or AT content [17]. There are two primary explanations for this bias: 1) a polymerase chain reaction (PCR) amplification bias, where high or low GC content reduces the efficiency of PCR amplification [18]; and 2) a reduced efficiency of capture probe hybridization to sequences with high or low GC content [19]. Whereas the former bias is inherent of the sequences to be amplified, the latter is a property of the capture probes, and may to some extent be compensated by probe design. To study the GC bias effect, we utilized density plots as described by Clark et al. [10], where we plotted GC content against the normalized mean read depth (Figure 5 and Additional file 2: Figure S2). All four technologies showed bias against very low $(<30 \%)$ and very high $(>70 \%)$ GC content. All the technologies, except Nextera, demonstrated a sharp fall in read depth for GC contents of $60 \%$ or higher. Nextera gave increased coverage for sequences with higher GC content, owing to the preference of the transposon technology used [20]. All the technologies gave poor coverage for sequences with less than $25 \% \mathrm{GC}$ content.

\section{Ability to detect SNVs}

An important goal of exome resequencing is to identify sequence variants. Therefore, we systematically compared the efficiency of exome capture for allele detection among the four technologies. We used UnifiedGenotyper, implemented in the GATK package [21], to investigate the 

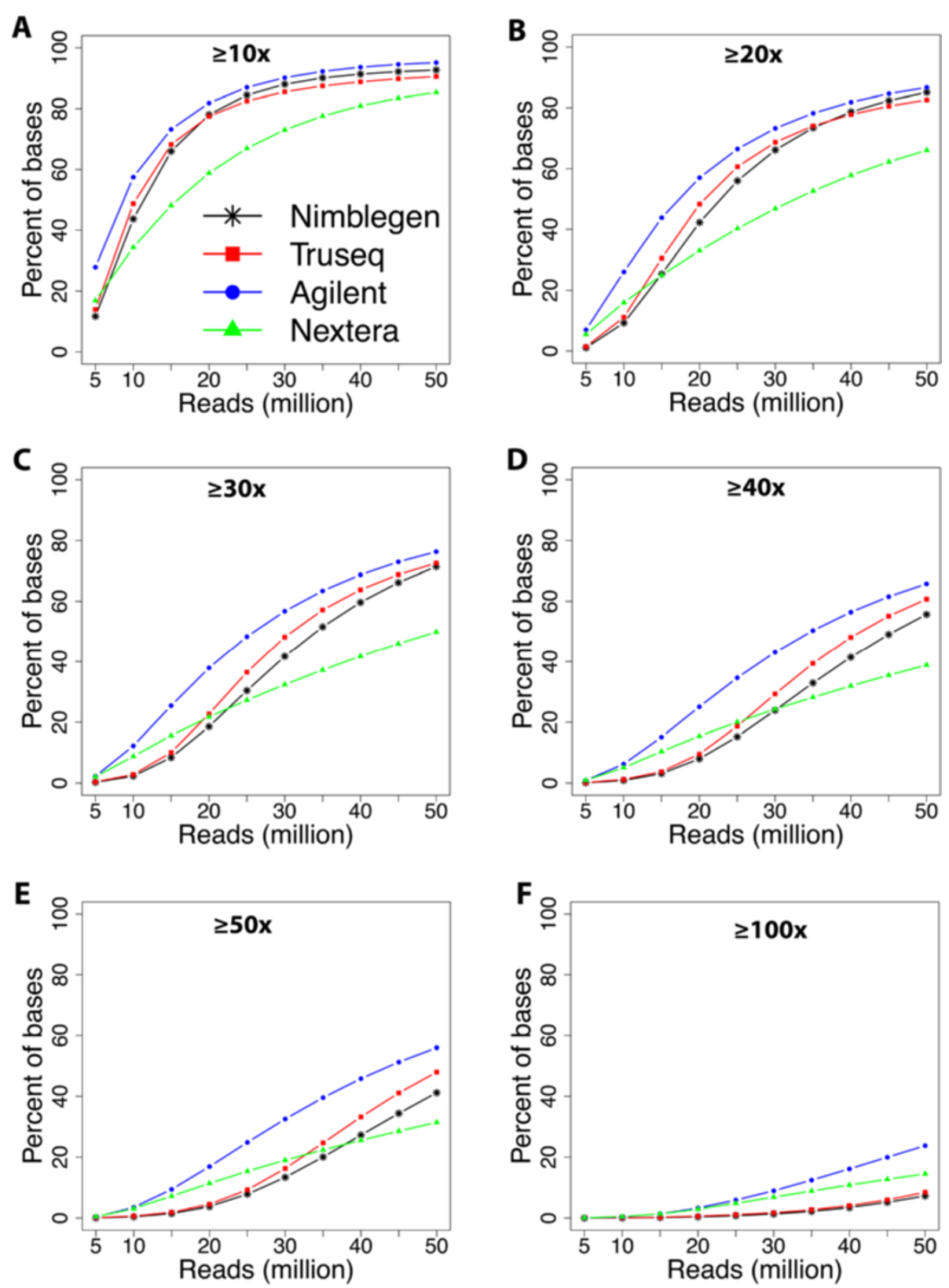

Figure 4 Coverage efficiency as a function of number of reads. The percent of targeted bases covered at $\mathbf{A}$ ) $\geq 10 x, \mathbf{B}) \geq 20 x, \mathbf{C}) \geq 30 \mathrm{x}$, D) $\geq 40 x, E) \geq 50 x$, and F) $\geq 100 x$ depths.

relationship between read counts and total single nucleotide variants (SNVs) detected within different intervals. As read counts increased, the number of SNVs identified in their target regions increased initially, and became saturated at approximately 20 million reads (Figure 6A). Very few additional SNVs were identified beyond 20 million reads. When considering the SNVs identified on their respective target regions, there is a clear correlation between the total number of SNVs detected and the number of bases targeted; NimbleGen detected the highest number of SNVs followed by TruSeq, Nextera, and Agilent (Figure 6A and Additional file 3: Figure S3A). A different trend was clear in the $26 \mathrm{Mb}$ region shared by all four technologies, where Agilent detected the highest number of SNVs, followed by Truseq, Nextera, and NimbleGen (Figure 6B and Additional file 3: Figure S3B). The majority of newly detected SNVs were common.

We also investigated SNV detection in the regions covered by the CCDS (Figure 6C), RefSeq (Figure 6D), and Ensembl (Figure 6E) exome databases. The Illumina technologies, TruSeq and Nextera, and NimbleGen detected similar number of SNVs in CCDS and RefSeq. However in 


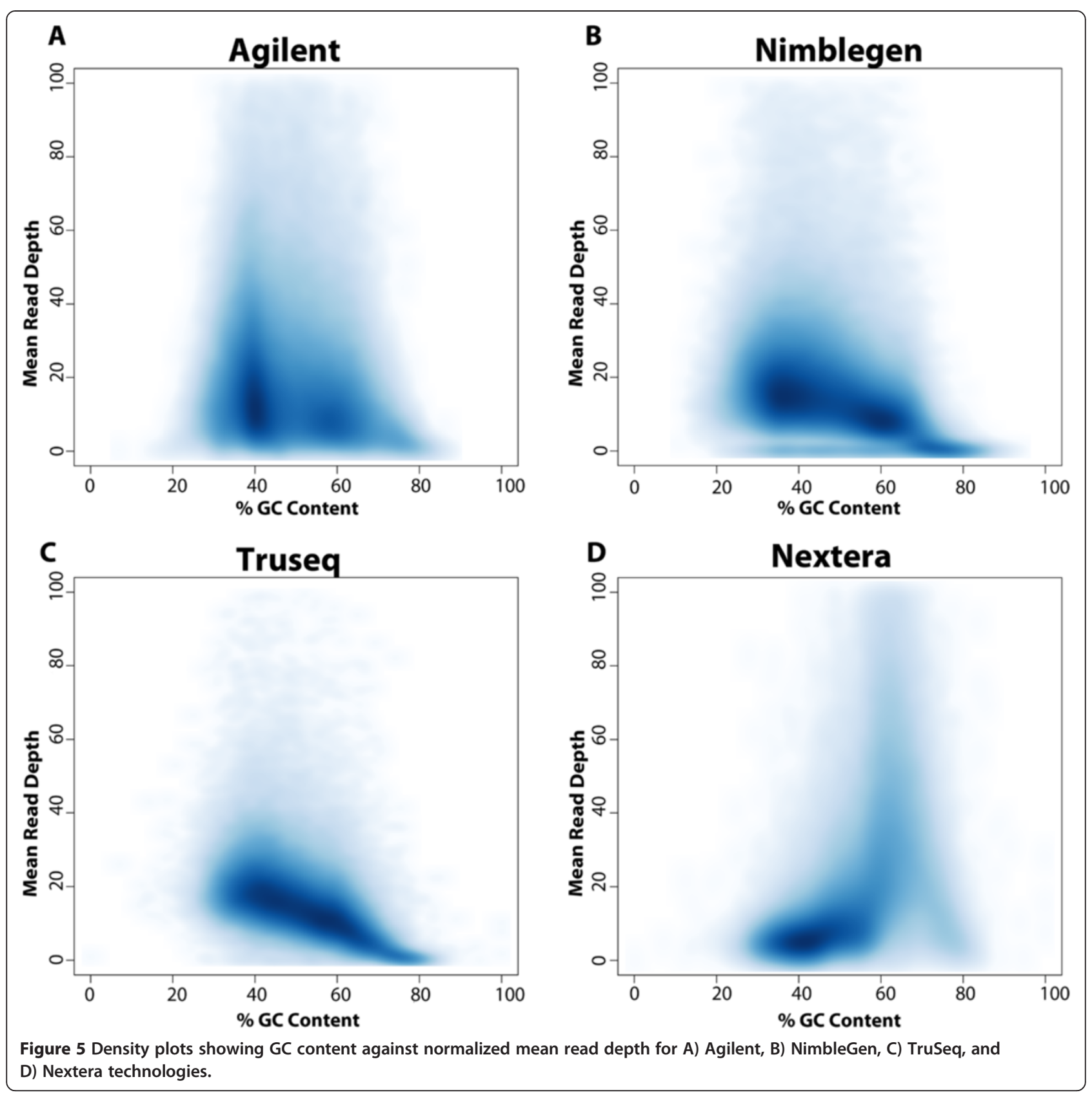

Ensembl regions, NimbleGen detected the highest number of SNVs. As expected, Illumina technologies detected a much larger number of SNVs in UTRs. Illumina technologies also covered the highest number of bases in the UTRs, followed by NimbleGen and Agilent (Figure 1F). Interestingly, at low read counts, more SNVs were detected by TruSeq, but at 40 million read counts, Nextera surpassed TruSeq.

We also investigated whether capture technologies showed bias in substitution detection, but none of the technologies showed bias towards specific nucleotide substitutions (Additional file 4: Figure S4 and Additional file 5:
Figure S5). Transitions were expected to occur twice as frequently as transversions. The transition-transversion (ts/tv) ratio is a metric for assessing the specificity of new SNP calls. We assessed the ts/tv ratio on their respective target regions (including non-exonic segments), and it ranged from 2.215 in Nextera to 2.257 in Agilent (Additional file 4: Figure S4). Previous studies have shown ts/tv ratios of $\approx$ 2.0-2.1 for whole genome datasets [22]. The Nextera and TruSeq technologies showed very similar ts/tv ratios, caused most likely by their identical target regions. Also, Agilent and NimbleGen had very similar ts/tv ratios. The difference in ts/tv ratios between Illumina technologies 

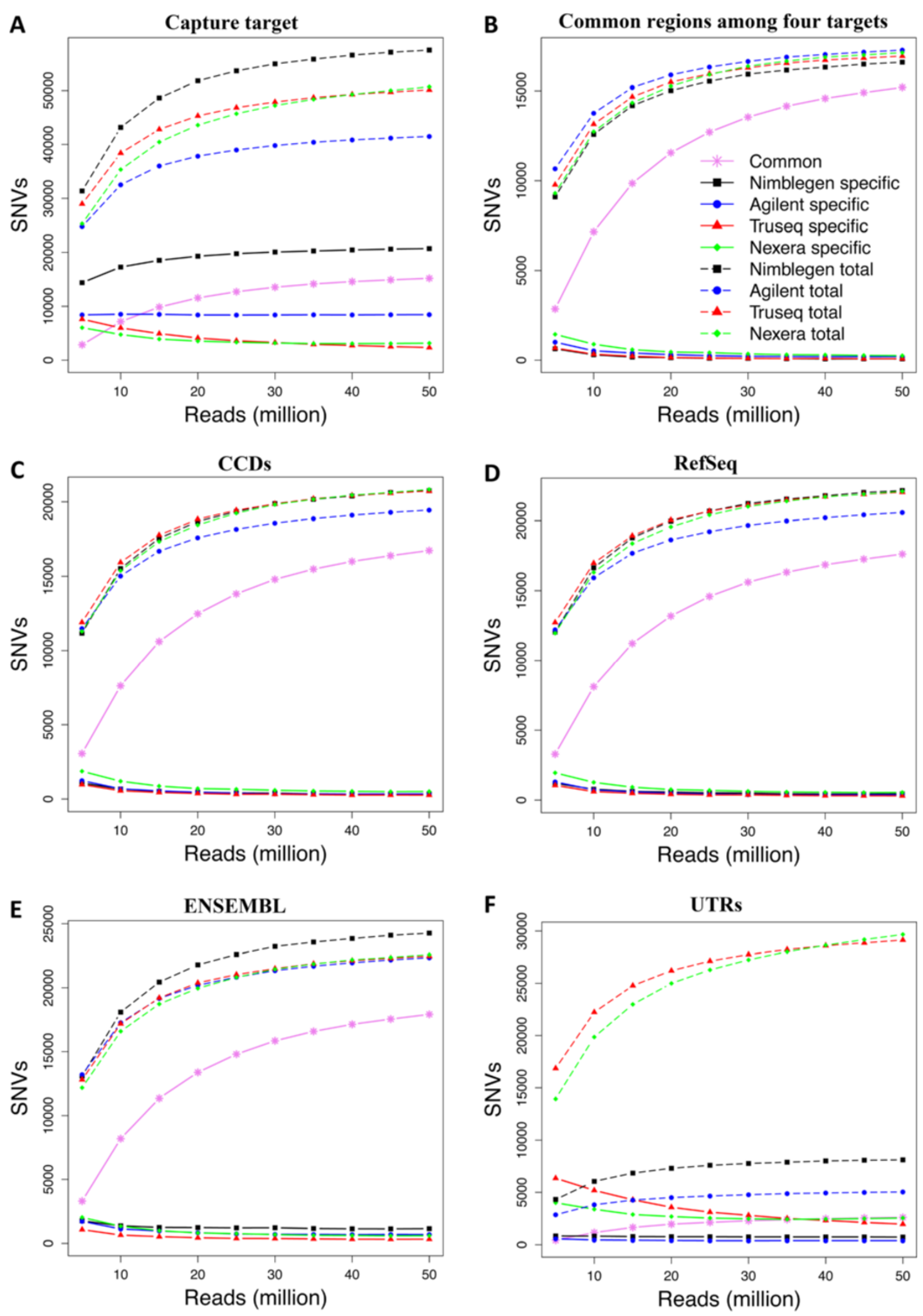

Figure 6 SNV detection by technology as a function of increasing read counts on A) intended target region, B) regions common among technologies, C) CCDS exons, D) RefSeq exons, E) Ensembl exons, and F) UTRs. Solid-lines indicate technology specific SNVs, dashed-lines indicate total number of SNVs, and solid pink lines indicate the SNVs common between the four technologies. 
(TruSeq and Nextera) and non-Illumina technologies (Agilent and NimbleGen) may be because Illumina technologies target a significantly higher number of UTRs than the other technologies. We also determined the ts/tv ratio in CCDS coding exons (Additional file 5: Figure S5). The ts/ tv ratio on CCDS ranges from 3.054 in Nextera to 3.109 in NimbleGen. It has been previously shown that the ts/tv ratio is $\approx 3.0-3.3$ for exonic variation [23].

\section{Detection of insertions and deletions}

Small insertions and deletions (indels) were called using the UnifiedGenotyper algorithm implemented in the GATK package [21]. Indel size ranged from -40 to +37 bases in Agilent, -61 to +37 bases in NimbleGen, -66 to +52 bases in TruSeq, and -66 to +90 bases in Nextera. Most indels were single bases, and more than $90 \%$ of the indels were less than seven bases long; this pattern was observed for all four technologies (Additional file 6: Figure S6A). At low read counts, TruSeq and NimbleGen detected a higher number of indels, followed by Nextera and Agilent (Figure 7A). At 15 million read counts, TruSeq surpassed NimbleGen, and at 20 million reads, Nextera surpassed Agilent (Figure 7A). Interestingly, at 50 million reads, Nextera surpassed NimbleGen (Figure 7A). At all the read counts, a disturbing fact was that very few indels were common across the four technologies, especially on CCDS, Ensembl and RefSeq regions.

Figure 7B shows a head-to-head comparison of indel detection in the regions covered by all four technologies. At all read counts, Agilent detected the highest number of indels. At lower read counts, NimbleGen detected more indels than TruSeq and Nextera; at 15 million reads, both Nextera and TruSeq surpassed NimbleGen. Only about $50 \%$ of indels were common among four technologies.

Indel detection in the regions covered by exome databases was also studied (Figure $7 \mathrm{C}-\mathrm{E}$ ). The number of indels detected in exons was significantly lower, than indels detected on the respective technology target regions and UTRs. We observed more indels of three or six bases (Additional file 6: Figure S6B), probably due to the negative selection of sizes not equal to multiples of three bases in coding sequences because they cause deleterious frame shift mutations.

When compared between replicates, both SNVs (Additional file 7: Figure S7 and Additional file 8: Figure S8) and indels (Additional file 9: Figure S9), showed similar trends in detecting total number of variants and showed very high overlap in newly detected variants.

\section{Discussion}

Continuous advancement in sequencing technologies increases the throughput of DNA sequencing, while at the same time contributes sharply to decreasing its cost.
Although sequencing costs have fallen, whole genome sequencing is still quite expensive, and data interpretation remains challenging. Therefore, whole genome sequencing is not the most appropriate choice for all investigations. The ability to target certain regions of the genome, such as protein and or RNA-coding exons, is an attractive alternative for many experiments. In recent times, target enrichment by hybridization technologies has demonstrated rapid progress in development and usage by the research and diagnostic community.

We present a comparative study of four whole exome capture technologies from three manufacturers, designed to reveal important performance aspects of the technologies. To address this, we studied six parameters for each technology: the portion of target bases representing different exome databases, target coverage efficiency, GC bias, sensitivity in SNV detection, sensitivity in small indel detection, and reproducibility.

Although all four exome capture technologies show very high target enrichment efficiency and cover large portions of the exome, only a small portion of the CCDS exome is uniquely covered by each technology (Figure 1C). Therefore, a researcher who is planning exome sequencing should assess which technology best covers the regions of interest to the investigation. Agilent targets the smallest part of the genome with $51.1 \mathrm{Mb}$, followed by Illumina technologies with $62.08 \mathrm{Mb}$, and NimbleGen with 64.1 Mb. There are 26.2 Mb of the human genome shared by all four technologies; the majority of which falls in CCDS exonic regions. Illumina not only encompasses far more UTRs, but also shows a higher coverage of RefSeq, CCDS, and Ensembl exome databases, followed by NimbleGen and Agilent.

Target coverage efficiency differs between the four technologies. Using pass-filter reads, Agilent shows higher coverage efficiency than the other technologies, which may be partially explained by the smaller targeted region $(51.1 \mathrm{Mb}$ ) compared with $64.1 \mathrm{Mb}$ and $62.08 \mathrm{Mb}$ for NimbleGen and Illumina respectively. Among the Illumina technologies, TruSeq gave a more uniform coverage than Nextera, but both had inferior efficiency compared with Agilent. Agilent gives the highest percentage of usable reads (pass-filter reads) (71.7\%), closely followed by NimbleGen.

Regardless of high or low target region GC content, there was a negative correlation between sequencing coverage and extreme GC content. Preference for transposon targets with high GC content can help explain non-uniform coverage for the Nextera technology.

Most researchers aiming for exome sequencing, especially in the medical sciences, focus on protein-coding regions. Therefore, the ability to identify SNVs and indels in coding regions is critical to many applications. 

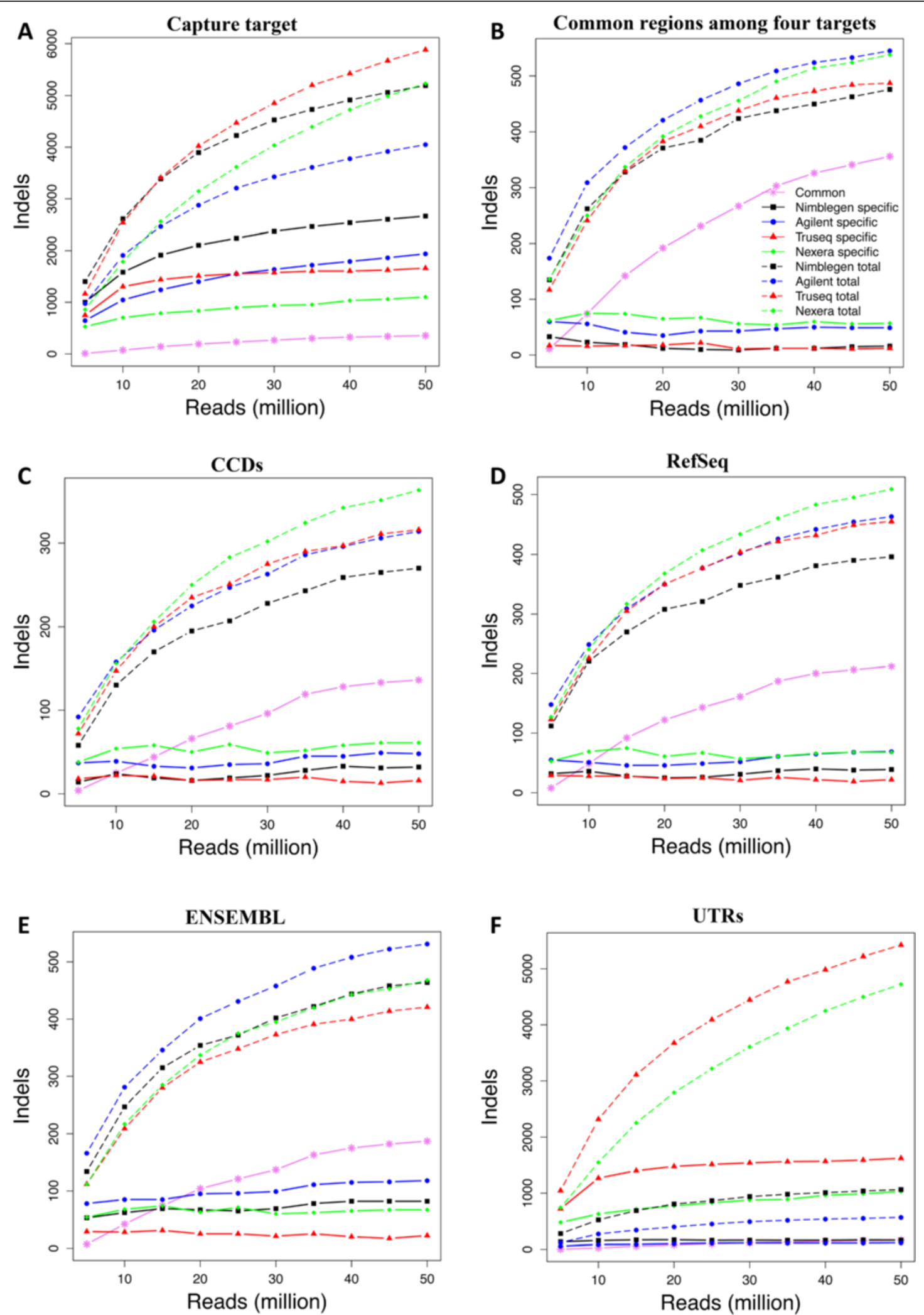

Figure 7 Indels detection by technology as a function of increasing read counts on A) intended target region, B) regions common among the technologies, C) CCDS exons, D) RefSeq exons, E) Ensembl exons, and F) UTRs. Solid-lines indicate technology specific SNVS, dashed-lines indicate total number of SNVs, and solid pink lines indicate the SNVs common between four technologies. 


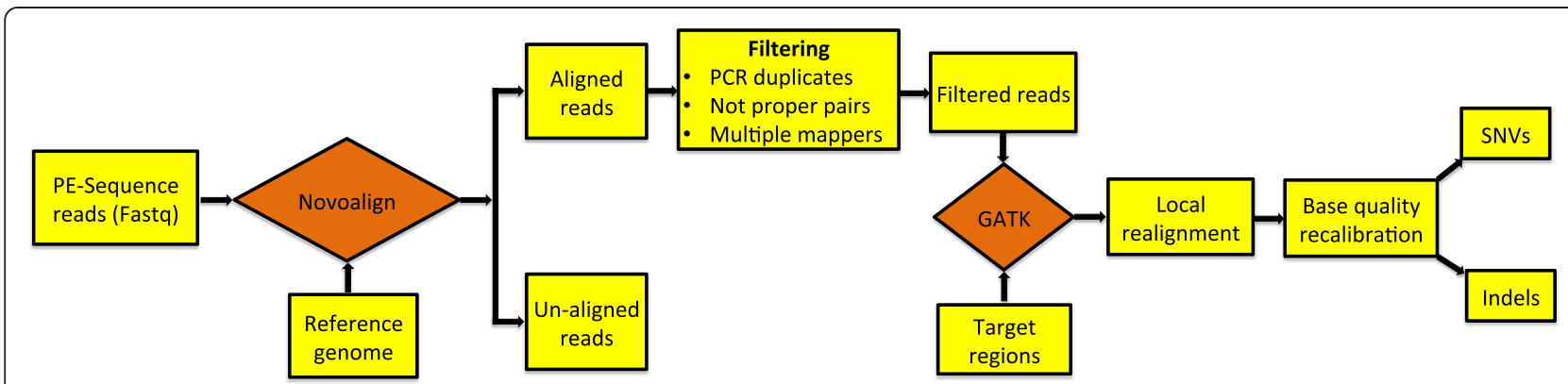

Figure 8 Overview of the computational pipeline.

NimbleGen captures the highest number of SNVs, followed by Illumina technologies and Agilent, when the total number of SNVs detected are correlated with technology target size. However, the number of bases sequenced also has cost and capacity considerations. Our results suggest that Illumina technologies detect a higher number of SNVs over the other technologies with regard to SNV detection against the CCDS and RefSeq exomes, owing to a higher coverage of these regions, but Agilent was better at detecting indels. We also observed that Nextera shows a clear edge over other technologies in the CCDS and RefSeq exomes, because it covers a larger fraction of these sequences.

We did not observe significant differences in technical reproducibility between the four technologies. However, we could, by comparing performance between replicates to the differences observed above, conclude that although some differences in SNV and indel detection were due to random experimental error, the major effect appears to be due to technological biases.

Since the comparison is based on a tumor sample, which may contains genomic aberrations that could differentially affect the performance of each technology, we investigated the coverage differences in COSMIC cancer genes. No significant deviation in coverage was observed when compared with global coverage (Figure 3 and Additional file 10: Figure S10).

Another important consideration is exome capture technologies evolve rapidly. For instance, Agilent recently released their next version of exome capture SureSelect Human All Exon V5. Although these versions do differ with regard to the genomic regions they target, about $84 \%$ of target region bases overlap. Illumina also has a new version, with a smaller targeted panel, just for exons. It is called Nextera Rapid Capture Exome (37 Mb), while the larger panel version is now named Nextera Expanded Exome (62 Mb). Illumina has also improved the Nextera protocol, with the Nextera Rapid kit; this improvement may reduce the $\mathrm{GC}$ bias observed here.
In total, our data suggest that all four technologies offer comparable performance. Other factors, such as the DNA content of the targeted regions, the amount of input DNA required, the extent of automation in library construction, and the cost of reagents to reach a certain depth of coverage, need to be considered before selecting the exome capture technology most appropriate for your particular application.

Readers should keep in mind that this study is based on one biological sample with two replicates. The observed technical reproducibility is very high and variability may be higher when two biological replicates are compared.

\section{Conclusions}

We systematically evaluated the performance of four whole exome capture technologies, and show that all the exome capture technologies perform well, but do exhibit consistent differences. Illumina covers a greater portion of coding exon bases across all the databases, followed by NimbleGen and Agilent. All the technologies give high coverage of their respective target regions, with the Agilent technology giving highest coverage (99.8\%) followed by Nextera (98.2\%), Truseq (96.9\%), and NimbleGen (96.5\%) of the intended targets. Nextera shows a sharp increase in read depth for GC content of $60 \%$ or higher compared other technologies. In common regions covered by all four technologies, Agilent detects slightly higher number of SNVs, followed by Nextera, TruSeq and Nimblegen. At all the read counts very few indels were common across the four technologies. All technologies give high technical reproducibility. One major limitation is that none of the capture technologies are able to cover all of the exons of the CCDS, RefSeq or Ensembl databases. Our study should help researchers who are planning exome sequencing experiments select the most appropriate technology for their study, without having to perform expensive and time-consuming comparisons. 


\section{Methods}

\section{Sample collection and library preparation}

One human osteosarcoma was selected from a tumor collection at the Department of Tumor Biology at the Norwegian Radium Hospital. The tumor was collected immediately after surgery after written informed consent, cut into small pieces, frozen in liquid nitrogen and stored at $-70^{\circ} \mathrm{C}$ until use.

High quality genomic DNA was isolated using the Promega Wizard Genomic DNA Purification Kit. One $\mu \mathrm{g}$ of genomic DNA was used to produce each exome captured sequencing library for four different technologies: NimbleGen SeqCap EZ v3.0, Agilent SureSelect XT2 Human All Exome v4.0, Illumina TruSeq Exome Enrichment kit and Illumina Nextera Exome Enrichment kit. The exome captured library preparation from the last three technologies was done following the manufacturers' protocols applying pre-capture multiplexing. The protocol for NimbleGen SeqCap EZ was adapted from the company's application note (http://www.nimblegen. com/products/lit/NimbleGen_SeqCap_EZ_SR_Pre-Captured_Multiplexing.pdf). The exome captured sequencing libraries were quality-controlled using an Agilent 2100 Bioanalyzer, and quantified using the Agilent QPCR NGS Library Quantification Kit (illumine GA) prior to cluster generation on an Illumina cBot.

\section{Datasets}

The human reference genome (hg19), RefSeq, CCDS, and Ensembl databases were downloaded from the UCSC genome table browser (http://genome.ucsc.edu/).

Because of Norwegian legal regulations, the ethical approval for this study and the consent signed by the patient, we are not able to deposit our dataset in a public repository. We will provide access to the data if requested.

\section{Sequencing and bioinformatics data analysis}

Sequencing of each exome capture library was done at the Oslo University Hospital Genomics Core Facility, using an Illumina HiSeq 2000 machine, as pair-end 100bp reads, following the manufacturer's protocols using TruSeq SBS v3. We developed an in-house pipeline for analysis, which integrates several existing programs (Figure 8).

Briefly, initial FASTQ files were subjected to quality control with the FastQC tool (http://www.bioinformatics.babraham. ac.uk/projects/fastqc/). Raw reads from each capture library were aligned to the human reference genome (hg19) with Novoalign (http://novocraft.com/), using default parameters. If more than one pair (PE sequencing) had identical start and end coordinates, they were considered PCR duplicates and were removed using in-house scripts. Filtered read counts were normalized to $50 \mathrm{M}$ reads between all four exome capture sequencing experiments by randomly selecting $50 \mathrm{M}$ reads from each filtered read set. These randomly selected sets were further used to select 5$50 \mathrm{M}$ reads, using an increment of $5 \mathrm{M}$ reads.

SNVs and indels were called with GATK [21]. The GATK pipeline was independently run on each data set. We followed the procedure recommended by the GATK documentation. Reads around indels were realigned. To remove systematic biases in quality scores, base quality score recalibration was done. The UnifiedGenotyper algorithm was run using a stand_emit_conf of 10.0 and stand_call_conf of 30.0. All variants with a Phred-based quality score $<30.0$ were called low quality and ignored.

\section{Additional files}

Additional file 1: Figure S1. Coverage efficiency shown as a bar plot for different depths for replicate 1.

Additional file 2: Figure S2. Coverage efficiency on high (>70\% GC) and low GC $(<30 \% \mathrm{GC})$ regions, as a function of number of reads. The percent of targeted bases covered at $\mathbf{A}) \geq 10 \times$ B) $\geq 20 \times \mathbf{C}$ ) $\geq 30 \times$ D) $\geq 40 \times$ E) $\geq 50 x$ and $\mathbf{F}$ ) $\geq 100 x$ depths.

Additional file 3: Figure S3. Comparison of SNVs detection by each technology at 50 million reads. A) SNVs detected on intended target regions, and $\mathbf{B})$ SNVs detected on regions shared by all four technologies.

Additional file 4: Figure S4. Mutation spectra by technology on intended target regions. Bar plots showing relative mutation frequency of different types of mutations for A) Agilent, B) NimbleGen, C) TruSeq, and D) Nextera technologies. Transition/Transversion (ts/tv) ratio indicated.

Additional file 5: Figure S5. Mutation spectra from CCDS exonic regions by technology. Bar plots show the relative mutation frequency of different types of mutations for A) Agilent, B) NimbleGen, C) TruSeq, and D) Nextera technologies. Transition/Transversion (ts/tv) ratio indicated.

Additional file 6: Figure S6. Indel size distribution by technology. A) Size distribution of all the indels that fall within the technology target regions. B) Size distribution of indels in CCDS coding exons.

Additional file 7: Figure S7. Comparison between two technical replicates in detecting SNVs, for A) Agilent, B) NimbleGen, C) TruSeq, and D) Nextera technologies. Smooth lines indicate SNVs detected on respective target regions, and dotted lines indicate SNVs detected on the target regions shared by all four technologies. Each figure shows the total number of SNVs detected by each replicate, common SNVS between replicates, and technology specific SNVs.

Additional file 8: Figure S8. Comparison of SNVs detection by each technology at 50 million reads on regions shared by all four technologies between two replicates for $\mathbf{A}$ ) Agilent, B) NimbleGen, C) TruSeq, and D) Nextera technologies.

Additional file 9: Figure S9. Comparison between two technical replicates in detecting indels, for $\mathbf{A})$ Agilent, B) NimbleGen, C) TruSeq, and D) Nextera technologies. Smooth lines indicate indels detected on intended target regions, and dotted lines indicate indels detected on the target regions shared by all four technologies. Each figure shows the total number of indels detected by each replicate, common indels between the replicates, and technology specific indels.

Additional file 10: Figure S10. Coverage efficiency comparison by technology on cancer genes. The smooth line indicates replicate 1 and the dotted line indicates replicate 2 .

\section{Abbreviations}

Bp: Base pair; CCDS: Consensus coding sequence; GATK: Genome analysis toolkit; indel: Insertion-deletion; miRNA: microRNA; SNP: Single nucleotide polymorphism; SNV: Single nucleotide variant; Mb: Megabase; ts: Transition; tv: Transversion; pcr: Polymerase chain reaction; UTRs: Untranslated regions. 


\section{Competing interests}

The authors declare that they have no competing interests.

\section{Authors' contributions}

LAMZ designed the study. SL and JS carried out experimental work. Computational analysis carried out by CCSR, MAM and DV. The manuscript was written by CCSR; SL and JS contributed experimental material and methods to manuscript. EH and OM participated in discussion and interpretation of the results. All the authors read, reviewed and approved the manuscript.

\section{Acknowledgments}

We would like to thank Jan Christian Bryn and Russell Castro for help with manuscript preparation. The Research Council of Norway (grant no. 218241/ $H I 0)$ and Radiumhospitalets legacy financially supported this study.

\section{Author details}

'Department of Tumor Biology, Oslo University Hospital, Norwegian Radium Hospital, 0310 Oslo, Norway. ${ }^{2}$ Department of Medical Informatics, Oslo University Hospital, Norwegian Radium Hospital, Oslo, Norway. ${ }^{3}$ Norwegian Cancer Genomics Consortium, Oslo, Norway. ${ }^{4}$ Genomics Core Facility, Oslo University Hospital, Oslo, Norway. ${ }^{5}$ Department of Informatics, University of Oslo, Oslo, Norway.

Received: 15 November 2013 Accepted: 27 May 2014

Published: 9 June 2014

\section{References}

1. Luikart G, England PR, Tallmon D, Jordan S, Taberlet P: The power and promise of population genomics: from genotyping to genome typing. Nat Rev Genet 2003, 4:981-994.

2. Yu TW, Chahrour MH, Coulter ME, Jiralerspong S, Okamura-lkeda K, Ataman B, Schmitz-Abe K, Harmin DA, Adli M, Malik AN, D'Gama AM, Lim ET, Sanders SJ, Mochida GH, Partlow JN, Sunu CM, Felie JM, Rodriguez J, Nasir RH, Ware J, Joseph RM, Hill RS, Kwan BY, Al-Saffar M, Mukaddes NM, Hashmi A, Balkhy S, Gascon GG, Hisama FM, LeClair E, et al: Using whole-exome sequencing to identify inherited causes of autism. Neuron 2013, 77:259-273.

3. Schuster B, Knies K, Stoepker C, Velleuer E, Friedl R, Gottwald-Muhlhauser B, de Winter JP, Schindler $D$ : Whole exome sequencing reveals uncommon mutations in the recently identified Fanconi anemia gene SLX4/FANCP. Hum Mutat 2013, 34:93-96.

4. Kalsoom UE, Klopocki E, Wasif N, Tariq M, Khan S, Hecht J, Krawitz P, Mundlos S, Ahmad W: Whole exome sequencing identified a novel zinc-finger gene ZNF141 associated with autosomal recessive postaxial polydactyly type A. J Med Genet 2013, 50:47-53.

5. Izumi R, Niihori T, Aoki Y, Suzuki N, Kato M, Warita H, Takahashi T, Tateyama M, Nagashima T, Funayama R, Abe K, Nakayama K, Aoki M, Matsubara Y: Exome sequencing identifies a novel TTN mutation in a family with hereditary myopathy with early respiratory failure. J Hum Genet 2013, 58:259-266.

6. Albert TJ, Molla MN, Muzny DM, Nazareth L, Wheeler D, Song X, Richmond TA, Middle CM, Rodesch MJ, Packard CJ, Weinstock GM, Gibbs RA: Direct selection of human genomic loci by microarray hybridization. Nat Methods 2007, 4:903-905.

7. Hodges E, Xuan Z, Balija V, Kramer M, Molla MN, Smith SW, Middle CM, Rodesch MJ, Albert TJ, Hannon GJ, McCombie WR: Genome-wide in situ exon capture for selective resequencing. Nat Genet 2007, 39:1522-1527.

8. Bainbridge MN, Wang M, Burgess DL, Kovar C, Rodesch MJ, D'Ascenzo M, Kitzman J, Wu YQ, Newsham I, Richmond TA, Jeddeloh JA, Muzny D, Albert TJ: Whole exome capture in solution with $3 \mathrm{Gbp}$ of data. Genome Biol 2010, 11:R62.

9. Marine R, Polson SW, Ravel J, Hatfull G, Russell D, Sullivan M, Syed F, Dumas M, Wommack KE: Evaluation of a transposase protocol for rapid generation of shotgun high-throughput sequencing libraries from nanogram quantities of DNA. Appl Environ Microbiol 2011, 77:8071-8079.

10. Clark MJ, Chen R, Lam HY, Karczewski KJ, Chen R, Euskirchen G, Butte AJ, Snyder M: Performance comparison of exome DNA sequencing technologies. Nat Biotechnol 2011, 29:908-914.

11. Sulonen AM, Ellonen P, Almusa H, Lepisto M, Eldfors S, Hannula S, Miettinen T, Tyynismaa H, Salo P, Heckman C, Joensuu H, Raivio T, Suomalainen A, Saarela J: Comparison of solution-based exome capture methods for next generation sequencing. Genome Biol 2011, 12:R94.
12. Asan, $X u Y$, Jiang $H$, Tyler-Smith $C$, Xue $Y$, Jiang $T$, Wang J, Wu M, Liu X, Tian G, Wang J, Wang J, Yang H, Zhang X: Comprehensive comparison of three commercial human whole-exome capture platforms. Genome Biol 2011, 12:R95.

13. Parla JS, lossifov I, Grabill I, Spector MS, Kramer M, McCombie WR: A comparative analysis of exome capture. Genome Biol 2011, 12:R97.

14. Pruitt KD, Tatusova T, Maglott DR: NCBI reference sequences (RefSeq): a curated non-redundant sequence database of genomes, transcripts and proteins. Nucleic Acids Res 2007, 35:D61-D65.

15. Flicek P, Amode MR, Barrell D, Beal K, Brent S, Carvalho-Silva D, Clapham P, Coates G, Fairley S, Fitzgerald S, Gil L, Gordon L, Hendrix M, Hourlier T, Johnson N, Kähäri AK, Keefe D, Keenan S, Kinsella R, Komorowska M, Koscielny G, Kulesha E, Larsson P, Longden I, McLaren W, Muffato M, Overduin B, Pignatelli M, Pritchard B, Riat HS, et al: Ensembl 2012. Nucleic Acids Res 2012, 40:D84-D90.

16. Pruitt KD, Harrow J, Harte RA, Wallin C, Diekhans M, Maglott DR, Searle S, Farrell CM, Loveland JE, Ruef BJ, Hart E, Suner MM, Landrum MJ, Aken B, Ayling S, Baertsch R, Fernandez-Banet J, Cherry JL, Curwen V, Dicuccio M, Kellis M, Lee J, Lin MF, Schuster M, Shkeda A, Amid C, Brown G, Dukhanina $\mathrm{O}$, Frankish A, Hart J, et al: The consensus coding sequence (CCDS) project: Identifying a common protein-coding gene set for the human and mouse genomes. Genome Res 2009, 19:1316-1323.

17. Gnirke A, Melnikov A, Maguire J, Rogov P, LeProust EM, Brockman W, Fennell T, Giannoukos G, Fisher S, Russ C, Gabriel S, Jaffe DB, Lander ES, Nusbaum C: Solution hybrid selection with ultra-long oligonucleotides for massively parallel targeted sequencing. Nat Biotechnol 2009, 27:182-189.

18. Aird D, Ross MG, Chen WS, Danielsson M, Fennell T, Russ C, Jaffe DB, Nusbaum C, Gnirke A: Analyzing and minimizing PCR amplification bias in Illumina sequencing libraries. Genome Biol 2011, 12:R18.

19. Kane MD, Jatkoe TA, Stumpf CR, Lu J, Thomas JD, Madore SJ: Assessment of the sensitivity and specificity of oligonucleotide (50mer) microarrays. Nucleic Acids Res 2000, 28:4552-4557.

20. Green B, Bouchier C, Fairhead C, Craig NL, Cormack BP: Insertion site preference of Mu, Tn5, and Tn7 transposons. Mob DNA 2012, 3:3.

21. McKenna A, Hanna M, Banks E, Sivachenko A, Cibulskis K, Kernytsky A, Garimella K, Altshuler D, Gabriel S, Daly M, DePristo MA: The Genome Analysis Toolkit: a MapReduce framework for analyzing next-generation DNA sequencing data. Genome Res 2010, 20:1297-1303.

22. Ebersberger I, Metzler D, Schwarz C, Paabo S: Genomewide comparison of DNA sequences between humans and chimpanzees. Am J Hum Genet 2002, 70:1490-1497.

23. Freudenberg-Hua Y, Freudenberg J, Kluck N, Cichon S, Propping P, Nothen MM: Single nucleotide variation analysis in 65 candidate genes for CNS disorders in a representative sample of the European population. Genome Res 2003, 13:2271-2276.

doi:10.1186/1471-2164-15-449

Cite this article as: Chilamakuri et al:: Performance comparison of four exome capture systems for deep sequencing. BMC Genomics 2014 15:449.

\section{Submit your next manuscript to BioMed Central and take full advantage of:}

- Convenient online submission

- Thorough peer review

- No space constraints or color figure charges

- Immediate publication on acceptance

- Inclusion in PubMed, CAS, Scopus and Google Scholar

- Research which is freely available for redistribution 